\title{
Nonlinear Equivalent Resistance-based Maximum Power Point Tracking (MPPT)
}

\author{
Chaitanya Poolla*, Abraham K. Ishihara ${ }^{\dagger}$ \\ ECE, Carnegie Mellon University (SV) \\ Moffett Field, CA 94035 \\ Email: *cpoolla@alumni.cmu.edu, †abe.ishihara@west.cmu.edu
}

\begin{abstract}
We present a nonlinear equivalent resistance tracking method to optimize the power output for solar arrays. Tracking an equivalent resistance results in nonlinear voltage step sizes in the gradient descent search loop. We introduce a new model for the combined solar module along with a DC-DC converter which results in a highly nonlinear dynamical system due to the inherent non-linearity of the PV cell topology and the switched DC-DC converter system. To guarantee stability over a range of possible operating regimes, we utilize a feedback linearization control approach to exponentially converge to the setpoint. Simulations are presented to illustrate the performance and robustness of the proposed technique.
\end{abstract}

Index Terms-Solar, Photovoltaic, Maximum Power Point Tracking, MPPT, Feedback Linearization, Buck-boost converter

\section{INTRODUCTION}

Recently, we have seen solar installations in the US more than double in every market segment [1]. Despite this recent growth, improvements in reliability testing, and advancements in solar cell efficiency, significant questions remain regarding the actual performance of PV modules in the field. Nonuniform changes in solar cell parameters may render modules more susceptible to hot-spot generation, especially under soiling and partial shading conditions. In order to optimize performance, per panel Maximum Power Point Tracking (MPPT) leveraging intelligent control techniques has been shown to be a viable solution [2].

The problem of Maximum Power Point Tracking (MPPT) has been well-studied in the literature [3]. Common approaches include, Hill-Climbing, Perturb and Observe $(\mathrm{P} \& \mathrm{O})$, Incremental Conductance (IC), Fuzzy Logic (FL), Neural Networks (NN), and Ripple Correlation Control (RCC). The non-model based approaches such as Hill-Climbing and $\mathrm{P} \& \mathrm{O}$ seek to estimate the sign of the gradient at the operating point on the P-V curve. Assuming uniform conditions without faults the P-V curve is known to have a single maximum. Hence, knowledge about the sign of the gradient is sufficient to determine the direction of perturbation in the voltage space. However, inappropriate choice of step sizes often lead to oscillations at or near the MPP. Incremental conductance-based approaches [4] approximate the slope along the P-V curve as $\frac{d P}{d V}=I+V \frac{\Delta I}{\Delta V}$. Thus, measurements of instantaneous and incremental conductance are sufficient to determine the direction of perturbation. While IC is capable of tracking changing weather conditions quickly, it can result in oscillations similar to $\mathrm{P} \& \mathrm{O}[5]$. On the other hand, sophisticated approaches such as those based on Fuzzy Logic or Neural Networks require regular tuning for adaptability. The tracking performance of FL-based approaches depend on the choice of membership functions [6]. In case of NN-based approaches, the network needs to trained for a given PV array and tuned further to adapt to changing array characteristics [3]. Further, the Maximum Power Point (MPP) of the PV system depends on local weather conditions and hence accurate prediction of the environmental conditions [7] enables better MPP tracking [8].

In this work, we consider a PV plant integrated with a buckboost converter supporting a load. The schematic is shown in Figure 2. By adjusting the duty cycle of the converter, the operating point of the PV system can be driven toward the MPP. Unlike several Hill-Climbing or P\&O approaches that seek to uniformly perturb the operating voltage, we propose to perturb the equivalent resistance (or, conductance) at the operating point which results in nonlinear voltage changes. This perturbation sets up the target operating point for the inner loop tracker, which is implemented in the buck-boost converter using feedback linearization. The main contribution of this paper is the combination of an equivalent resistance tracking outer-loop based on a feedback linearization innerloop control law that takes into account the highly nonlinear plant dynamics.

The rest of the paper is structured as follows: Section III provides an overview of the outer-loop iterative update along with the derivation of closed-form equations using the Lambert-W function in Section [II-A The model of the PV module integrated with a buck-boost converter along with corresponding dynamics is described in Section III The feedback linearization controller is derived in Section IV Simulation results are presented in Section $\mathrm{V}$ along with conclusions in Section VI

\section{Nonlinear Equivalent Resistance Tracking}

The effective or equivalent resistance seen by the solar module is given by

$$
R_{e q}=\frac{V}{I}
$$

where $V$ and $I$ are the operating voltage and current of the module, respectively. Let us denote the operating point at time $t_{k} \in \mathbb{R}^{+}$by the pair $\left(I_{k}, V_{k}\right) \in \mathbb{R}^{2}$. Given an operating point $\left(I_{k}, V_{k}\right)$, the goal of the outer-loop MPPT algorithm is 
to determine a new operating point at time $t_{k+1}$ given by $\left(I_{k+1}, V_{k+1}\right)$ such that $I_{k+1} \cdot V_{k+1}>I_{k} \cdot V_{k}$. If the algorithm converges, then the solar module will be operating at a local maximum of the power curve. Under uniform conditions, there is only one global maximum and hence, gradient descent algorithms will converge to the maximum power point.

A challenge is to determine change $\left(\Delta I_{k}, \Delta V_{k}\right) \stackrel{\text { d }}{=}$ $\left(I_{k+1}, V_{k+1}\right)-\left(I_{k}, V_{k}\right)$ since the power versus voltage (or current) landscape is highly nonlinear. It is common to use a constant $\Delta V>0$ voltage increment or decrement depending on the slope of the power versus voltage curve, $\left.\frac{d P}{d V}\right|_{\left(I_{k}, V_{k}\right)}$. If $\left.\frac{d P}{d V}\right|_{\left(I_{k}, V_{k}\right)}>0, V_{k+1}=V_{k}+\Delta V$. If $\left.\frac{d P}{d V}\right|_{\left(I_{k}, V_{k}\right)}<0$, $V_{k+1}=V_{k}-\Delta V$. The problem with this approach is that a constant $\Delta V$ that works in one region of the I-V curve may not work in another. For example, a small $\Delta V$ increment that is suitable to the left of the MPP, may be too large of an increment for operating regimes to the right of the MPP due to the large negative slope of the $I-V$ curve.

An alternative approach is to consider a constant equivalent resistance (or, conductance) change when the operating point is in region I (or, II) (Fig. 11). If the operating point is in region I, we increase the equivalent resistance by a constant $\Delta R_{e q}$.

$$
R_{e q_{k+1}}=R_{e q_{k}}+\Delta R_{e q}
$$

where $\Delta R_{e q}>0$ is a constant for all $t_{k}$. Using (1), (2) becomes

$$
\frac{V_{k+1}}{I_{k+1}}=\frac{V_{k}}{I_{k}}+\Delta R_{e q} \stackrel{\mathrm{d}}{=} \beta_{k}
$$

When the operating point of the solar module is in region II (Fig. 11), we choose to increment by the inverse of equivalent resistance, or equivalent conductance. That is:

$$
\frac{I_{k+1}}{V_{k+1}}=\frac{I_{k}}{V_{k}}+\Delta G_{e q} \stackrel{\mathrm{d}}{=} \frac{1}{\beta_{k}}
$$

It again follows that (compare to 3 )

$$
V_{k+1}=\beta_{k} I_{k+1}
$$

Given $\beta_{k}$ (Equations 3 or 4, we may use the one-diode model of the solar array and the Lambert-W function [9, 10] to compute the new (perturbed) operating poin 1 as summarized below.

\section{A. Outer-Loop Update using Lambert-W function}

In the simplest representation, the PV cell is assumed to be a superposition of the dark and illuminated current-voltage characteristics. Along with the series and shunt resistances, the I-V relationship of the PV cell is described here. In what follows, $I_{p h}(\mathrm{~A})$ denotes the photo-generated current, $I_{0}(\mathrm{~A})$ denotes the dark saturation current, $q$ (Coulombs) denotes the electric charge carried by a single photon, $k$ denotes the Boltzmann constant $\left(J \cdot K^{-1}\right)$, and $T$ denotes the cell temperature $(K)$. Let us consider the following equation $\mathrm{s}^{2}$ to model the $\mathrm{I}-\mathrm{V}$ characteristics of the PV cell:

$$
V=\beta I
$$

\footnotetext{
${ }^{1} \mathrm{~A}$ two diode model can also be used [11]

${ }^{2}$ For the sake of notational convenience, we omit the subscripts $k$ from $\beta_{k}$ and $k+1$ from $V_{k+1}$ and $I_{k+1}$ in Equation 5
}

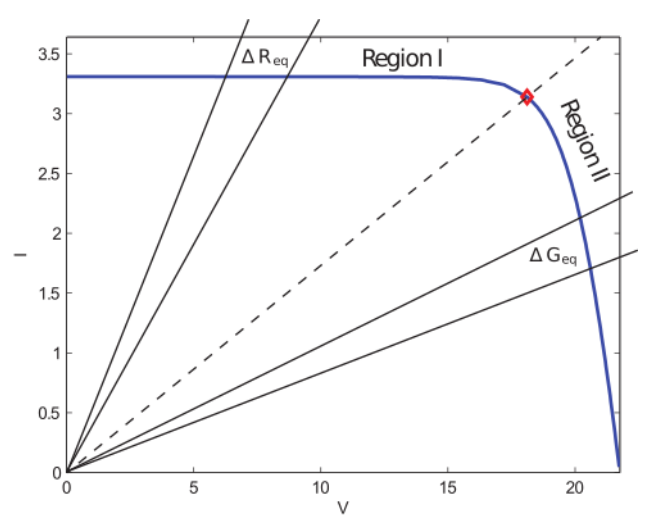

Fig. 1: Regions I and II of I-V curve

and

$$
I=I_{p h}-I_{01}\left(e^{\frac{\left(V+R_{s} I\right)}{V_{T_{1}}}}-1\right)-\frac{V+I R_{s}}{R_{s h}}
$$

Substitution of (6) into (7) yields:

$$
V=c_{0}^{-1} \beta\left(I_{p h}+I_{01}-I_{01} e^{\left(\frac{\beta+R_{s}}{\beta V_{T}}\right)} V\right)
$$

where

$$
c_{0}=1+\frac{\beta+R_{s}}{R_{s h}}
$$

Equation $(8)$ is of the form

$$
y=d_{0}-d_{1} e^{\alpha_{1} y}
$$

where

$$
\begin{aligned}
d_{0} & =c_{0}^{-1} \beta\left(I_{p h}+I_{01}\right) \\
d_{1} & =c_{0}^{-1} \beta I_{01} \\
\alpha_{1} & =\frac{\beta+R_{s}}{\beta V_{T}}
\end{aligned}
$$

We can transform 9 to $W e^{W}=x$ where

$$
\begin{aligned}
W & =\alpha_{1} d_{0}-\alpha_{1} y \\
x & =\alpha_{1} d_{1} e^{\alpha_{1} d_{0}}
\end{aligned}
$$

Using the Lambert-W function [10], we can obtain closed-form solutions with $y=V$ as follows: $y=$ $\frac{1}{\alpha_{1}}\left(\alpha_{1} d_{0}-W(x)\right)$. The implication is that, given $\beta_{k}$ and the $\mathrm{I}-\mathrm{V}$ parameters, the new (perturbed) operating point can be exactly computed. This enables one to rapidly simulate electrical performance under the time-varying non-uniform conditions. The presented approach above is scalable, robust, and readily extends to arbitrary circuit topologies. Given $N_{p}$ strings of $N_{s}$ cells connected in series per string, the single cell parameters $\left(I_{p h}, I_{01}, R_{s}, R_{s h}, V_{T}\right)$ scale into the corresponding module parameters $\left(N_{p} I_{p h}, N_{p} I_{01}, \frac{N_{s}}{N_{p}} R_{s}, \frac{N_{s}}{N_{p}} R_{s h}, N_{s} V_{T}\right)$.

\section{Modeling Solar Module and DC-DC Buck BoOst CONVERTER WITH PARASITIC LOSSES}

We consider a dynamical model of the PV-Buck-Boost system with parasitic resistances in the inductor, capacitor, and 


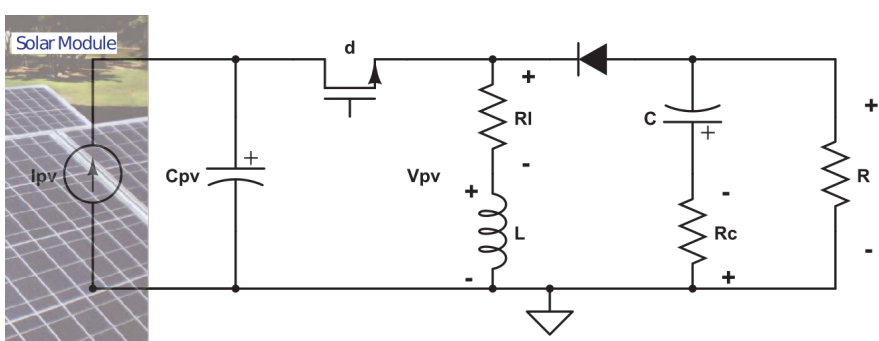

Fig. 2: Solar Module and DC-DC Buck Boost Converter with Parasitic losses.

the on-state of the MOSFET. The circuit diagram is shown in Fig. 2 .

By representing the circuit elements as equations, we derive the dynamics of the system below.

Switch-On Model: When the switch is in the on-state, the equations become:

$$
\begin{aligned}
\frac{d}{d t}\left[\begin{array}{c}
v_{p v} \\
v_{c} \\
i_{L}
\end{array}\right]= & {\left[\begin{array}{ccc}
0 & 0 & -\frac{1}{C_{p v}} \\
0 & -\frac{1}{C\left(R+R_{C}\right)} & 0 \\
\frac{1}{L} & 0 & -\frac{\left(R_{o n}+R_{L}\right)}{L}
\end{array}\right]\left[\begin{array}{c}
v_{p v} \\
v_{c} \\
i_{L}
\end{array}\right] } \\
& +\left[\begin{array}{c}
\frac{I_{p v}}{C_{p v}} \\
0 \\
0
\end{array}\right] \\
= & A_{1} x+h_{1}(x)
\end{aligned}
$$

Switch-Off Model: When the switch is in the off-state, the equations become:

$$
\begin{aligned}
\frac{d}{d t}\left[\begin{array}{c}
v_{p v} \\
v_{c} \\
i_{L}
\end{array}\right]= & {\left[\begin{array}{ccc}
0 & 0 & 0 \\
0 & -\frac{1}{C\left(R+R_{C}\right)} & \frac{R}{C\left(R+R_{c}\right)} \\
0 & -\frac{R}{L\left(R+R_{C}\right)} & -\frac{\left(R_{L}+R_{d}+R_{c} \| R\right)}{L}
\end{array}\right]\left[\begin{array}{c}
v_{p v} \\
v_{c} \\
i_{L}
\end{array}\right] } \\
& +\left[\begin{array}{c}
\frac{I_{p v}}{C_{p v}} \\
0 \\
-\frac{V_{D}}{L}
\end{array}\right] \\
= & A_{2} x+h_{2}(x)
\end{aligned}
$$

Averaged Model: Using the averaged circuit model approach, the switched linear system can be approximate ${ }^{3}$ by a single nonlinear system given by

$$
\dot{x}=A(x, d)+d h_{1}+(1-d) h_{2}
$$

$A(x, d) \stackrel{\mathrm{d}}{=} d A_{1}+(1-d) A_{2}=\left[\begin{array}{ccc}0 & 0 & -\frac{d}{C_{p v}} \\ 0 & -\frac{1}{C\left(R+R_{C}\right)} & \frac{(1-d) R}{C\left(R+R_{C}\right)} \\ \frac{d}{L} & -\frac{(1-d) R}{L\left(R+R_{C}\right)} & a_{33}\end{array}\right]$

and $a_{33}=-\frac{1}{L}\left\{d\left(R_{o n}+R_{L}\right)+(1-d)\left(R_{L}+R_{d}+R_{C} \| R\right)\right\}$. In the above, $\left(v_{p v}, v_{c}, i_{L}\right)$ are the state variables, $d \in[0,1]$ is the control (duty-cycle) and the nonlinearities are due to the multiplicative control and state terms and the nonlinear function of the state variable: $I\left(v_{p v}\right)$.

\footnotetext{
${ }^{3}$ The degree to which the nonlinear system approximates the switched linear system can be measured by application of the Baker-Campbell-Hausdorff formula.
}

\section{FEEDBACK LineARIZATION CONTROL}

For controller design we consider (12) but without parasitic resistances. However, in the simulation presented in Section $\mathrm{V}$, the controller is found to be robust even in the presence of parasitic resistances. The dynamics without the parasitic resistances are given by:

$$
\left[\begin{array}{c}
\dot{v}_{p v} \\
\dot{v}_{c} \\
i_{L}
\end{array}\right]=\left[\begin{array}{ccc}
0 & 0 & -\frac{d}{C_{p v}} \\
0 & -\frac{1}{C R} & \frac{1-d}{C} \\
\frac{d}{L} & -\frac{1-d}{L} & 0
\end{array}\right]\left[\begin{array}{c}
v_{p v} \\
v_{c} \\
i_{L}
\end{array}\right]+\left[\begin{array}{c}
\frac{I_{p v}}{C_{p v}} \\
0 \\
0
\end{array}\right]
$$

In the following, we discuss only the mechanics of the feedback linearization controller design. We do not discuss the stability nor robustness properties. Readers interested in the theory should consult [12].

Region I Controller: Consider an operating point in region I as shown in Fig. 11 We assume a reference equivalent resistance is generated via (2) denoted by $R_{e q_{r e f}}$. In order to ensure tracking of $R_{e q_{r e f}}$ we define the output variable:

$$
y_{R_{e q}}(x)=R_{e q_{r e f}}-R_{e q}(x)
$$

Differentiating $y_{R_{e q}}(x)$ with respect to time, we obtain

$$
\dot{y}_{R_{e q}}(x)=-\frac{d}{d t}\left(R_{e q}(x)\right)=-\frac{I_{p v} \dot{v}_{p v}-v_{p v} \dot{I}_{p v}}{I_{p v}^{2}}
$$

Application of the chain rule yields:

$$
\dot{I}_{p v}=\frac{d}{d t} I_{p v}\left(v_{p v}\right)=\frac{\partial I_{p v}}{\partial v_{p v}} \dot{v}_{p v}
$$

Consider $N_{s}$ cells in series with identical parameters. If we have $N_{p}$ strings of $N_{s}$ cells each, in parallel, then

$$
I_{p v}=I_{p h}-I_{01}\left(e^{\frac{v_{p v}+R_{s} I_{p v}}{V_{T}}}-1\right)-\frac{v_{p v}+R_{s} I_{p v}}{R_{s h}}
$$

where,

$$
\begin{gathered}
I_{p h}=N_{p} I_{p h}^{(c)} \\
I_{01}=N_{p} I_{01}^{(c)} \\
R_{x}=\frac{N_{s}}{N_{p}} R_{s}^{(c)} \\
R_{s h}=\frac{N_{s}}{N_{p}} R_{s h}^{(c)} \\
V_{T}=N_{s} V_{T}^{(c)}
\end{gathered}
$$

Thus we have,

$$
\begin{gathered}
\frac{\partial I_{p v}}{\partial v_{p v}}=-\frac{I_{01}}{V_{T}} e^{\frac{v_{p v}+R_{s} I_{p v}}{V_{T}}}\left(1+R_{s} \frac{\partial I_{p v}}{\partial v_{p v}}\right) \\
-\frac{1}{R_{s h}}\left(1+R_{s} \frac{\partial I_{p v}}{\partial v_{p v}}\right) \\
\Longrightarrow \frac{\partial I_{p v}}{\partial v_{p v}}\left(1+\frac{I_{01} R_{s}}{V_{T}} e^{\frac{v_{p v}+R_{s} I_{p v}}{V_{T}}}+\frac{R_{s}}{R_{s h}}\right) \\
=-\frac{I_{01}}{V_{T}} e^{\frac{v_{p v}+R_{s} I_{p v}}{V_{T}}}-\frac{1}{R_{s h}}
\end{gathered}
$$


Hence we get,

$$
\frac{\partial I_{p v}}{\partial v_{p v}}\left(I_{p v}, v_{p v}\right)=-\frac{R_{s h} I_{01} e^{\frac{v_{p v}+R_{s} I_{p v}}{V_{T}}}+V_{T}}{V_{T} R_{s h}+I_{01} R_{s} R_{s h} e^{\frac{v_{p v}+R_{s} I_{p v}}{V_{T}}}+V_{T} R_{s}}
$$

We may now evaluate Equation 14

$$
\begin{aligned}
\dot{y} & =-\frac{1}{I_{p v}^{2}}\left(I_{p v}-v_{p v} \frac{\partial I_{p v}}{\partial v_{p v}}\right) \dot{v}_{p v} \\
& =-\frac{1}{I_{p v}^{2}}\left(I_{p v}-v_{p v} \frac{\partial I_{p v}}{\partial v_{p v}}\right)\left(-\frac{d}{C_{p v}} i_{L}+\frac{I_{p v}}{C_{p v}}\right) \\
& =-k y
\end{aligned}
$$

Setting $\dot{y}=-k y$ where $k>0$ and solving for $d$, we have

$$
\begin{aligned}
-\frac{d}{C_{p v}} i_{L}+\frac{I_{p v}}{C_{p v}} & =\frac{k y I_{p v}^{2}}{I_{p v}-v_{p v} \frac{\partial I_{p v}}{\partial v_{p v}}} \\
\frac{d i_{L}}{C_{p v}} & =\frac{I_{p v}}{C_{p v}}-\frac{k y I_{p v}^{2}}{I_{p v}-v_{p v} \frac{\partial I_{p v}}{\partial v_{p v}}} \\
& =\frac{I_{p v}\left(I_{p v}-v_{p v} \frac{\partial I_{p v}}{\partial v_{p v}}\right)-k y I_{p v}^{2} C_{p v}}{C_{p v}\left(I_{p v}-v_{p v} \frac{\partial I_{p v}}{\partial v_{p v}}\right)} \frac{C_{p v}}{i_{L}} \\
\Longrightarrow d & =\frac{I_{p v}\left(g\left(v_{p v}\right)-k y I_{p v} C_{p v}\right)}{i_{L} g\left(v_{p v}\right)}
\end{aligned}
$$

where, $g\left(v_{p v}\right)=I_{p v}-v_{p v} \frac{\partial I_{p v}}{\partial v_{p v}}$

Region II Controller: Consider an operating point in region II as shown in Fig. 11 In this case, we assume a reference equivalent conductance is generated via (4) denoted by $G_{\text {eq }}$. In order to ensure tracking of $G_{e q_{r e f}}$ we define the output variable:

$$
y_{G_{e q}}(x)=G_{e q_{r e f}}-G_{e q}(x)
$$

Proceeding as above, we may solve for the feedback linearizing control law that guarantees the output error converges exponentially to the origin.

Complete Control Law: We combine the results of region I and II into the following globally valid control law:

$$
d_{f l c}= \begin{cases}\frac{1}{i_{L}}\left(I_{p v}-\frac{k y_{R_{e}} I_{p v}^{2} C_{p v}}{g\left(v_{p v}\right)}\right) & \text { if Region I } \\ \frac{1}{i_{L}}\left(I_{p v}+\frac{k y_{G_{e}} v_{p v}^{2} C_{p v}}{g\left(v_{p v}\right)}\right) & \text { if Region II }\end{cases}
$$

\section{Simulation Results}

In this section we simulate the proposed feedback linearization-based control algorithm obtained in Equation 25 on the PV-buckboost platform. The electrical parameters of a Kyocera PV module $\left(N_{s}=36, N_{p}=1\right)$ employed are provided here: $R_{s}=0.01(\mathrm{Ohms}), R_{s h}=150(\mathrm{Ohms})$, $I_{01}=1.9795 \cdot 10^{-10}(\mathrm{~A})$, and $I_{p h}=3.31(\mathrm{~A})$. For the DC-DC Buck-Boost converter, the following parameters are used: $C=220 e^{-6}(F), L=3 e-3(H), R=10(\Omega)$, and $C_{p v}=1 e-3(F)$. We take the sampling period of the buckboost converter to be $T_{s}=1 / 100000(s)$. Under the standard test conditions, the module characteristics are shown in Figure 3 The MPP is achieved when the operating voltage is at
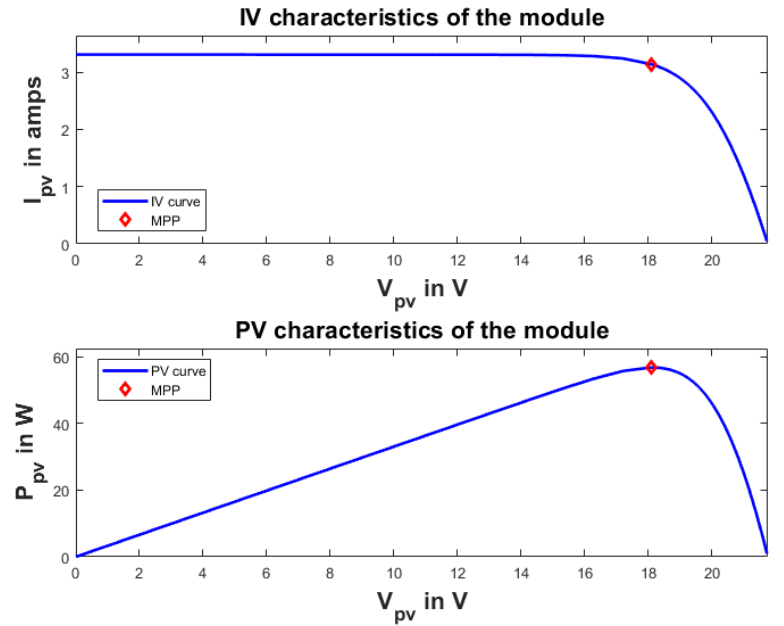

Fig. 3: I-V and P-V characteristics of the PV module considered.
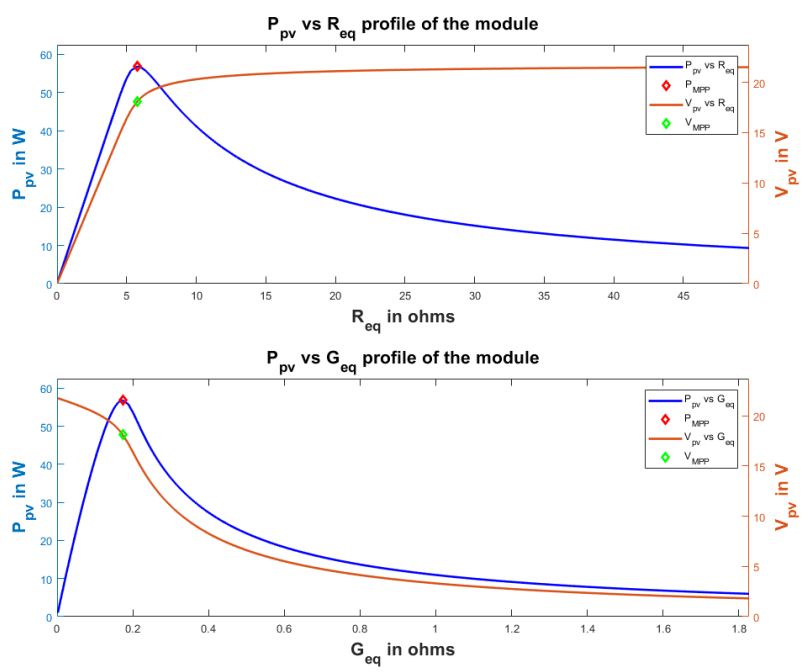

Fig. 4: $R_{e q}$ and $G_{e q}$ profiles of the PV module in relation to $P_{p v}$ and $V_{p v}$

$V_{m p p} \approx 18.1 \mathrm{~V}$. Therefore, region 1 can be understood to span the interval $\left[0, V_{m p p}\right)$ and region 2 would span the interval $\left[V_{m p p}, V_{o c}\right]$. The equivalent resistance for region 1 (and conductance for region 2) can be seen from Figure 4 The magnitude of $R_{e q}$ in region 1 and the magnitude of $G_{e q}$ in region 2 can be obtained from the portions of the graph that are left to the MPP. These magnitudes can provide cues to determine the step sizes associated with the iterative outer loop updates. Further, in order to adapt convergence based on the proximity to the MPP, the perturbation step sizes $\left(\Delta R_{e q}\right.$ and $\Delta G_{e q}$ ) are chosen to be proportional to estimated slope $\frac{\partial P_{p v}}{\partial V_{p v}}$. In this work, the PV-buckboost model was simulated in MATLAB with the above parameters for different initial conditions. The feedback linearization-based control converged to the correct Maximum Power Point by tracking the outerloop set points iteratively without prior knowledge of the 


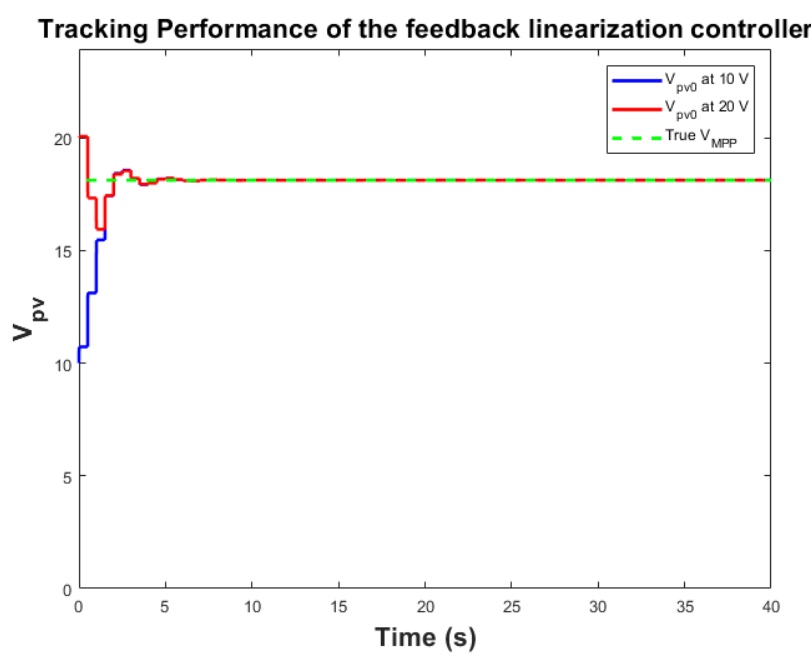

Fig. 5: Performance of Feedback Linearization Controller given in 25 for two initial conditions.

MPP. In order to test for robustness, we first note that the feedback controller derived in 24 does not account for parasitic resistances $\left(R_{c}, R_{L}, R_{o n}, R_{d}\right)$. However, for this simulation the values of $\left(R_{c}, R_{L}, R_{\text {on }}, R_{d}\right)$ were set to $(1,1,1,1000)$. The simulated trajectories starting from two initial conditions $\left(V_{p v}=10 \mathrm{~V}, V_{p v}=20 \mathrm{~V}\right)$ are depicted in Figure 5. It can be noted that the system trajectory converges to the MPP even in the presence of parasitic effects, demonstrating the robustness of the controller. Further, the choice of step sizes proportional to the estimated slope of the PV curve ensures that the system trajectory does not oscillate about the MPP.

\section{CONCLUSION}

This work presented a novel method for MPPT by a combination of an equivalent resistance tracking mechanism achieved by feedback linearization of buck-boost converter dynamics. The analytical determination of the outer-loop set points using the Lambert-W function is discussed based on PV diode models. The buck-boost dynamics are derived for different switch positions and the average dynamics is formulated. A feedback linearization-based control law is derived to track the reference signal. The adaptive outer-loop set points and the exponential inner-loop tracking ensures rapid convergence to the maximum power point of the solar module. Simulation results are presented with different initial conditions. Results indicate that the proposed approach enables robust and stable tracking. Future work will investigate the comparison of existing techniques to the proposed approach under time-varying changes in plant parameters in simulation and experiment.

\section{REFERENCES}

[1] US Solar Market Insight. "Year End Review Executive Summary". In: SEIA, Washington, DC, USA 2 (2011).
[2] Chris Deline et al. "A Performance and Economic Analysis of Distributed Power Electronics in Photovoltaic Systems A Performance and Economic Analysis of Distributed Power Electronics in Photovoltaic Systems". In: Energy 303.January (2011).

[3] Trishan Esram and Patrick L. Chapman. "Comparison of Photovoltaic Array Maximum Power Point Tracking Techniques". In: IEEE Transactions on Energy Conversion 2 (2007), pp. 439-449.

[4] K. Hussein et al. "Maximum photovolatic power tracking: An algorithm for rapidly changing atmospheric conditions". In: Proc. Inst. Elect. Eng. 142 (1995), pp. 59-64.

[5] YM Tung, Aiguo Patrick Hu, and Nirmal-Kumar Nair. "Evaluation of micro controller based maximum power point tracking methods using dSPACE platform”. In: Australian university power engineering conference. 2006.

[6] Mummadi Veerachary, Tomonobu Senjyu, and Katsumi Uezato. "Neural-network-based maximum-powerpoint tracking of coupled-inductor interleaved-boostconverter-supplied PV system using fuzzy controller". In: IEEE Transactions on Industrial Electronics 50.4 (2003), pp. 749-758.

[7] C. Poolla and A. K. Ishihara. "Localized solar power prediction based on weather data from local history and global forecasts". In: 2018 IEEE 7th World Conference on Photovoltaic Energy Conversion (WCPEC) (A Joint Conference of 45th IEEE PVSC, 28th PVSEC 34th EU PVSEC). June 2018, pp. 2341-2345. DOI: 10.1109/ PVSC.2018.8548173.

[8] Xianwen Gao, Shaowu Li, and Rongfen Gong. "Maximum power point tracking control strategies with variable weather parameters for photovoltaic generation systems”. In: Solar Energy 93 (2013), pp. 357-367.

[9] G. Petrone, G. Spagnuolo, and M. Vitelli. "Analytical model of mismatched photovoltaic fields by means of Lambert W-function," in: Sol. Energy Mater. Sol. Cells 91 (2007), pp. 1652-1657.

[10] R M Corless et al. "On the Lambert W function". In: Advances in Computational Mathematics 5.4 (1996), pp. 329-359. URL: http : / / dx . doi . org / 10 . 1007/ BF02124750.

[11] Abraham K Ishihara and Shahar Ben-Menahem. "Asymptotic Analysis of the Two-Diode Photovoltaic Model”. In: IASTED Power and Energy Systems and Applications (2011).

[12] R. Su, G. Meyer, and L.R. Hunt. Robustness in nonlinear control in 'Differential geometric control theory'. Birkhauser Boston, Cambridge, Mass. USA, 1983. 